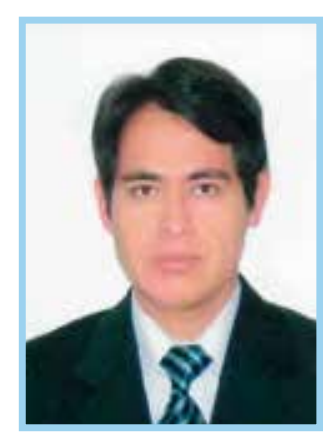

Walter Lizandro Arias Gallegos

\section{CRISIS DE LA \\ UNIVERSIDAD EN EL PERÚ: UN PROBLEMA DE SU NATURALEZA E IDENTIDAD}

\section{RESUMEN}

En el presente artículo se hace una reflexión sobre la identidad de las universidades católicas a partir de su naturaleza como institución eminentemente académica y de la crisis que se remite a la educación superior de nivel universitario. Se revisan aspectos históricos, las funciones de investigación, enseñanza y extensión de la universidad, así como los problemas de la educación universitaria que se comprenden la escasa producción académica, la deficiente enseñanza, su limitada conexión con la realidad del país y poca claridad en los roles de los profesores universitario y sus estudiantes.

\section{PALABRAS CLAVE}

Educación universitaria, investigación, identidad, Universidad Católica.

\section{ABSTRACT}

In this article, there is a reflection about the identity of Catholic Universities from its nature as an eminently academic institution and the crisis that is sent to the higher education of university level.

The historic aspects, research functions, teaching, university extension are reviewed, as well as the university education problems that comprise the poor academic production, deficient education, its limited connection with the reality of the country and little clarity on the functions of university teachers and their students.

\section{KEYWORDS}

University education, research, identity, Catholic University.

\section{INTRODUCCIÓN}

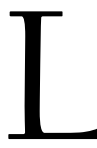

a universidad es una de las instituciones más antiguas de la humanidad. Su origen data del siglo X cuando empezaron a surgir las asociaciones gremiales. Así tenemos que la primera universidad surgió en 1088 en Bolonia, luego le siguieron la de París en 1150, la de Oxford en 1167, la de Padua en 1222, la de Salamanca en 1218, la de Viena en 1365, la de Praga en 1348, etc. (Greenwood, 2011). Asimismo, es importante tener en cuenta que las universidades surgieron como asociaciones de personas que tenían vivo interés por el conocimiento. Estas personas se reunían en las escuelas catedralicias como una comunidad de maestros y estudiantes (universitas magistrorum et scholarium) que trataba de escapar de la jurisdicción del obispo y apelaban directamente al Papa. 
De hecho, la primera universidad que se crea oficialmentefuela de París, al recibir sus estatutos en 1215 por parte del Cardenal Roberto de Courcon y quefueron ratificados en 1231 mediante la bula papal Parens Scientiarum (Hubeñak, 2008). De ahí que exista cierta confusión en las fechas de creación de las universidades, ya que algunos consideran la fecha en que comenzaron a reunirse los maestros y estudiantes, y otros consideran la fecha en que el Papa otorga la bula que legitima su funcionamiento.

Por otro lado, si bien las universidades surgen de la Iglesia, poco a poco se fueron desprendiendo de ella. Este fue un proceso lento y paulatino que inicia a finales del siglo XIV durante la alta escolástica, que continúa en el renacimiento, se agudiza en la edad moderna y se inserta culturalmente a través de la laicización de las instituciones sociales en el siglo de las luces (siglo XVIII). En ese sentido, en la escolástica, lo más característico de la universidad medieval era la disputatio, es decir, un duelo académico entre dos personas, sean estudiantes o profesores, en el que se discutían diversos temas con argumentos lógicos tomados de la filosofía y la teología. Se trataba de una confrontación de ideas que capturabalaatención de la comunidad académica, dependiendo de quiénes eran los participantes. Entre las disputas más memorables figura la de Pedro Abelardo y Bernardo de Claraval, de las que resultaba ganador siempre el primero.

El conocimiento que se desarrollaba reflejaba una unidad entre la fe y la razón, principalmente por influencia de Santo Tomás de Aquino que había conseguido integrar el conocimiento, tanto de la tradición greco-latina como de la filosofía islámica. En el renacimiento empero, por las ideas de Duns Scoto, Guillermo de Occam y Giordano Bruno; la fe y la razón vuelven a tomar caminos separados. La enseñanza pierde su énfasis teológico y se nutre de las aproximaciones racionales y empiristas de los filósofos modernos, de modo que el distanciamiento entre la enseñanza universitaria y la fe se hace más evidente.
Durante la Ilustración, se difunden diversas filosofías abiertamente anticlericales que promueven la teología protestante. El modelo de universidad de la época prescinde de la fe y comienza un proceso de laicización que implicó la creación de diversas escuelas y universidades a cargo del Estado. Otras tantas universidades católicas, fueron perdiendo su fidelidad para con la Iglesia y se sumaron al naturalismo liberal que promovía la razón ilustrada y el positivismo naciente. Entonces, la teología y la metafísica fueron arrancadas del conocimiento científico, que decantó en un naturalismo en el siglo XIX, y en un relativismo en la siguiente centuria.

Este cientificismo naturalista no ubica más el conocimiento en el plano sobrenatural y transmite esta visión hueca del mundo en el claustro universitario. La reforma napoleónica terminó por consolidar esta visión del mundo en diversas instituciones sociales, incluyendo las universidades que pasaban por un proceso de laicización. Es en este contexto que surge la Universidad de Berlín, como un nuevo modelo de universidad, en el que la investigación científica es la fuente del conocimiento superior (Perkins, 1967). Desde entonces la enseñanza universitaria ha privilegiado más, el conocimiento surgido de la investigación científica, positiva y naturalista; que el conocimiento filosófico, teológico y metafísico; que ha alongado aún más la brecha entre ciencia y fe.

Las universidades latinoamericanas, reproducirían estas tensiones entre la fe y la razón, pero de manera más lenta, dadas las condiciones geográficas y las limitaciones tecnológicas de la época. Según Molina (2008) la primera universidad latina se inauguró en 1538 en Santo Domingo, República Dominicana. Luego, en 1551 se crearon universidades en Lima y México, con diferencia de unos pocos meses. Para esa época en Europa sólo había 16 universidades y ninguna se había creado en EEUU todavía. Las universidades latinas eran copia de las Pontificias Universidades de Salamanca y Alcalá de Henares, 
donde predominaba por aquellos años, el modelo aristotélico-tomista.

En el siglo XIX, el siglo de las revoluciones latinoamericanas había ya 31 universidades con fuerte influencia del modelo napoleónico, que implicó la laicización de la enseñanza universitaria. Sin embargo, las universidades latinas, estaban inmersas en un ambiente politizante demasiado cargado, que conllevaría a la reforma de Córdova de 1918. Esta reforma critica el carácter aristocrático y elitista con que venían funcionando las universidades en el cono sur del continente (Lazo, 2002). Esta reforma supuso cambios drásticos en el sistema de organización de la enseñanza universitaria. Se implantan entonces medidas como la asistencia libre, la libertad de cátedra, la participación del estudiante en el gobierno de la universidad, la extensión universitaria y la autonomía universitaria. También significó el reemplazo de la enseñanza escolástica por la investigación científica.

Esta reforma se extendió por diversos países de la patria grande, en Chile tuvo lugar en 1922, en México en 1921, en Cuba en 1923, en Colombia en 1924 y en el Perú en 1926. Todo ello trajo consigo el crecimiento de la matrícula universitaria, el incremento de nuevas carreras, la pérdida de posición de algunas carreras tradicionales como el derecho, la diversificación de especialidades, el cambio de facultades a departamentos, el interés por la producción científica, la temporalidad de los cursos se volvió semestral y la enseñanza se tornó laica (Molina, 2008).

Para nuestro país, la creación de universidades inicia con la Universidad Nacional Mayor de San Marcos, creada el 12 de mayo de 1551, pero se inició el año académico el lro. de enero de 1553. Casi cien años después, en 1622 se crea la Universidad de San Ignacio de Loyola en el Cuzco pero se cierra en 1767 , fecha en que los jesuitas son expulsados del país. En 1677 se crea la Universidad San Cristóbal de Huamanga. Más tarde se crea en el Cuzco la Universidad
San Antonio Abad en 1692, y en 1824 se crea la Universidad de Trujillo (Aranzamendi, 2008). La Universidad Nacional de San Agustín se crea en Arequipa en 1828 por iniciativa de Dean Valdivia (Arenas, 1996). En 1917 el Padre Jorge Dintilhac de los Sagrados Corazones funda la Universidad Católica del Perú, que en 1942, el Papa Pío XII otorgaría el título de Pontificia (Cardó, 2005).

Más recientemente, vemos que con el Decreto Legislativo № 882 para la Promoción de la Inversión en la Educación, las universidades en el país han tenido un crecimiento acelerado, con la salvedad de que han proliferado sobre todo, "universidades negocio", cuyo rasgo más esencial es la visión de la universidad como una empresa (Castro y Valdivia, 2012).

Esto ha agudizado la crisis educativa del país, que tiene ya larga data y que es resultado de la incoherencia entre el discurso y el valor que le atribuimos a la educación en todos los niveles (Aranzamendi, 2008). Paraelcasodelaeducación universitaria, esto se manifiesta en una enseñanza que no responde a los tiempos que nos toca vivir ni a la situación socioeconómica que atraviesa el Perú. Vemos que se ha distorsionado totalmente, el fin de la universidad y que la sociedad misma demanda de ella la simple "formación" de personas con las capacidades que confiere el título, de ejercer una profesión; convirtiendo a las universidades en meras fábricas de títulos profesionales (Céspedes, 1994).

Renovar la visión de la universidad donde se alberga la masa crítica de académicos que se compromete con el desarrollo del país, a través de la generación de conocimientos científicos, tecnológicos, artísticos, filosóficos y teológicos; pasa por la revisión de la naturaleza de la universidad y la valoración concreta de la crisis que le eclipsa. Todo ello decanta en la forja de la identidad de la universidad, no como un negocio, sino como un espacio donde la élite académica se congrega con el principal afán de comprender la realidad, de modo que las diversas facetas de la vida, la sociedad y el espíritu humano se integren 
de manera armoniosa y nos permitan resolver los problemas sociales que afectan a las poblaciones menos favorecidas.

\section{NATURALEZA DE LA UNIVERSIDAD}

Comoyahabíamosmencionado,lasuniversidades fueron creadas con el fin de profundizar en el conocimiento de la realidad, y aunque existen diversas instituciones de educación de nivel superior, launiversidad sedistingueprecisamente por eso. En ese sentido, la Ley General de Educación 23384 contempla como formas de educación superior, la educación técnica (propia de Institutos Superiores Tecnológicos y Pedagógicos) y la universitaria. En los institutos se imparte formación técnica, que permite a los estudiantes desarrollar habilidades para operar máquinas y herramientas sobre la base de información tecnológica; mientras que en las universidades se debe generar conocimientos de alto nivel a través de la investigación científica.

Así pues, la Ley Universitaria № 23733, dice en su artículo 18 que "las funciones de la universidad son la formación profesional, la difusión cultural, la creación intelectual y artística, y la investigación científica y tecnológica”. Todo ello se resume en la investigación, la enseñanza y la extensión; el trípode que sostiene a toda universidad que se precie de ser tal (Perkins, 1967).

Sin embargo, lo más característico de la universidad es la investigación, que se convierte en el eje en torno al que giran la enseñanza y la extensión. Esto es así, porque si no hubiera investigación, los profesores no tendrían qué enseñar, o en todo caso la enseñanza impartida sería muy similar a la que se da en un contexto escolar, que es fruto de la indagación documental del maestro, pero no de la aplicación del método científico. Asimismo, si no hubiera investigación no habría actividades orientadas al desarrollo social, porque éstas se derivan de los avances científicos y tecnológicos que aporta la investigación.
Lamentablemente, la mayoría de universidades de nuestro país se limitan a la función de enseñanza sin considerar que la docencia sin investigación empobrece a la universidad y la transforma solo en un vehículo de transmisión de conocimientos y no en el centro de creación del saber (Delgado y Alcázar, 2004).

Como dice Reynoso (1998), si la integración de la enseñanza y la investigación es superficial e insustancial, es decir sin un norte definido o los soportes -humanos y sistémicosque garanticen su continuidad, se generan estructuras pedagógicas estériles que no guardan correspondencia con las exigencias sociales del desarrollo del país.

La enseñanza así es una mera abstracción. Por ello, es importante no perder de vista que una universidad que no genera conocimiento, brinda en realidad una enseñanza "escolarizada" y por tanto solo se desvirtúa a sí misma. Las universidades de mayor prestigio tienen muy claro este principio, de hecho, las mejores universidades del mundo son aquellas que están a la vanguardia del saber científico: cuentan con laboratorios y tecnología de punta, tienen un cuerpo de profesores que investigan a tiempo completo, su producción académica es además de vasta, de alto nivel y cuentan con políticas de investigación que promueven el desarrollo de todas estas actividades.

De acuerdo con el ranking de las doscientas mejores universidades del mundo elaborado por el diario británico The Times, las mejores universidades están en Estados Unidos, seguidas de las de Europa, Australia, Japón y China. En este ranking, la Universidad Nacional Autónoma de México -ila única latinoamericana!- se ubica en el puesto 195. Otro ranking de la Universidad Jiao Tong de Shangai señala que las ocho mejores Universidades del mundo están en Estados Unidos, entre ellas, la de Harvard, la de Berkeley, la de Stanford, el MIT, etc. 
En América Latina las mejores universidades se ubican en Brasil, como son la Universidad de Sao Paulo y la Universidad de Campinhas (Oppenheimer, 2008).

Esta situación está empezando a hacer eco en algunas autoridades políticas y académicas del país, que se ve reflejado en una preocupación por mejorar el posicionamiento de las universidades peruanas en los rankings nacionales e internacionales.

Los rankings nacionales de hace poco menos de una década señalaban que las mejores universidades eran la PUCP, la Universidad Peruana Cayetano Heredia, la Universidad Nacional MayordeSan Marcos, la Universidaddel Pacífico, la Universidad Nacional de Ingeniería, la Universidad Nacional Agraria La Molina, la Universidad del Altiplano, la Universidad Nacional de Trujillo y la Universidad Nacional de San Agustín (Piscoya, 2006).

Hoy en día los rankings identifican que las mejores universidades siguen siendo la PUCP, la UPCH, la UNMSM, la Universidad del Pacífico, la UNI y la Universidad Nacional Agraria La Molina; pero a estas se han agregado, la Universidad de Lima, la Universidad de Piura, la Universidad San Antonio Abad y la Universidad San Martín de Porres.

Este ranking toma en consideración el tamaño de la universidad (medido a través de la cantidad de carreras que oferta, la cantidad de profesores y estudiantes), la internacionalización, la empleabilidad de sus egresados, el prestigio que posee y las investigaciones publicadas.

La investigación es entonces el motor de la vida universitaria, que debe animar la enseñanza. $\mathrm{Al}$ respecto se debe tener presente que, si bien la universidad cumple también funciones formativas, por historia y tradición, estas son secundarias a la investigación.
En nuestro medio en cambio, no se reconoce este aspecto como parte inherente de la naturaleza de la universidad. Se toma lo formativo como primario y le secunda la investigación. Esta inversión de prioridades no permite ver el alcance que tiene la investigación en la enseñanza, y termina por debilitarla. Así pues, en el imaginario de la gente, no se ve la universidad como una institución eminentemente académica, sino como una fábrica de profesionales, no interesa cuanto se investiga, sino cuantos profesionales egresan. Por otro lado, quienes estudian en la universidad, responden a este modelo, de manera que los estudiantes no buscan aprender sino aprobar, no desean formarse para contribuir con el desarrollo de la nación, sino que solo quieren su título para conseguir un trabajo.

La educación universitaria, vista así, está mercantilizada. La oferta y la demanda son los ejes sobre los que gira la universidad y ya no el conocimiento. Las business university son precisamente este modelo de universidad al que nos referimos. En ellas no pesan los ideales intelectuales que toda universidad cultiva sino los conceptos funcionales que caracterizan a las empresas (Castro y Valdivia, 2012), por tanto no puede considerarse del mismo modo a una universidad que a una empresa (Casales, 2005). Así que, si bien muchas universidades son empresas privadas, tienen que regirse por fórmulas equilibradas que velen por la calidad y pertinencia de sus funciones primordiales (Ruíz, 2013).

Cuando la investigación antecede a la enseñanza la currícula tiene ciertos componentes, como la integración de los conocimientos, la investigación como pilar del desarrollo científico-tecnológico y la promoción de valores (Ferro, 2001); pero siempre de cara a la realidad más próxima. Por ello, Sime (2001) indica que los aspectos que influyen en la elaboración del currículo universitario son los factores temporales, disciplinarios, científicos, tecnológicos, ideológico-políticos, generacionales, las características de los estudiantes y del mercado. 
Ello supone que se tiene que prestar especial atención a las necesidades que determinadas sociedades demandan en circunstancias particulares. En el Perú por ejemplo, dados nuestros medios de producción, las profesiones altamente tecnologizadas no tendrían un espacio laboral adecuado.

Otro detalle que vale la pena tomar en cuenta es el perfil de los estudiantes que egresan de los colegios, ya que muchos de ellos no cuentan con la base académica suficiente para prolongar sus estudios en la universidad, sino que ven la universidad como una alternativa para ascender socialmente. En ese sentido, se sabe que el acceso a la educación superior constituye un mecanismo seguro para escapar de la pobreza en nuestro país (Castro, Casavilca y Lizarzaburu, 2010).

Un estudio publicado recientemente por el Instituto de Estudios Peruanos, reportó que un individuo con educación superior tiene un $70 \%$ de probabilidades de ascender socialmente (Benavides y Etesse, 2012). Esto explica por qué la gente acude cada vez con mayor interés a las universidades, muy a pesar de que este espacio no es compatible con sus aptitudes y su vocación. Téngase presente que en 1940 sólo el $1 \%$ de la población mayor de 15 años tenía educación superior, mientras que el año 2007 este porcentaje fue de $31 \%$.

Lo que la gente pasa por alto es que, cuando se estudia por la motivación equivocada, de no entrar al cauce propio del estudio universitario, el resultado solo puede ser el fracaso académico. Esto no quiere decir que los universitarios no deban desear una mejora en su calidad de vida, mediada por su paso por la universidad, sino que esto más bien viene por añadidura, siempre que demuestren una preocupación académica en sus estudios que pueda traducirse en competencia profesional en su trabajo, cuando se hayan titulado. En resumen, a la universidad debe ir gente que quiere estudiar, aprender y conocer; ya que esa es la naturaleza de la universidad.
Ahora bien, dado que los estudiantes no buscan esto de primera mano, es importante que las dimensiones curriculares comprendan la investigación, la docencia, la evaluación y la asesoría-tutoría (Sime, 2001), ya que en la medida que la universidad entregue a la sociedad, profesionales bien preparados, cumple con su misión social.

En ese sentido, la investigación y la enseñanza no pueden estar desvinculadas de la sociedad, sino que tienen que responder a ella. El binomio investigación-enseñanza en la universidad también requiere de una sociedad que le encuentre utilidad, así como las aplicaciones del conocimiento deben ser sujetadas por la investigación y la enseñanza (Perkins, 1967). De esta manera se cierra un círculo virtuoso que define y distingue a las universidades como verdaderas instituciones que enarbolan los estandartes del conocimiento, la cultura y el bienestar social.

Ubicada en el marco de la sociedad, la universidad no es una institución aislada, ya que muy por el contrario debe tener en el Estado y la empresa privada, aliados estratégicos que le permitan concretar sus metas. Las universidades que apuntan en esta dirección, consiguen amalgamar la ciencia, la tecnología y la sociedad (CTS) en campos como la investigación, la política, la educación y la economía. Esta nueva fórmula es la que a través de dos vertientes, ha facilitado el despegue de las universidades de Estados Unidos y Europa.

Por un lado, la tradición europea de la CTS parte de la sociología científica que toma a Kuhn como máximo representante, entre otros como Barry Barnes, Steven Shapin y David Bloor. Mientras que la tradición estadounidense se centra más en las consecuencias sociales de la ciencia y tiene como representantes a Paul Durbin, Ivan Illich, Carl Matcham y Langdon Winner. A pesar de sus diferencias, la CTS se sostiene en principios que promueven la interdisciplinariedad y 
responden a una imagen crítica, no reductiva, ni descontextualizada de la universidad (Quintero, 2010).

En este paradigma, la ciencia y la universidad son un instrumento del desarrollo tanto económico, como social y personal. En este escenario, la sociedad (representada por el Estado y la empresa) es un buen soporte para la investigación universitaria, pero su aporte retorna a ella a través de los logros de la ciencia y la tecnología. Bunge (1981) plantea en ese sentido que, las naciones que se cierran a este esquema son las más empobrecidas y culturalmente subdesarrolladas. ¿El Perú se encuentra en esta situación?

Para responder esta pregunta, sería bueno aclarar, que si bien el país muestra un crecimiento sostenible, desde hace un par de décadas aproximadamente; el boom turísticogastronómico y de la minería es hoy, lo que fue años atrás, la fiebre del caucho o del guano; y como todos sabemos, en ninguno de esos casos, el país consiguió quebrar las cadenas del subdesarrollo, antes bien, se engendraron más condiciones de pobreza y explotación, mediadas por la corrupción. Es decir, que si la economía nacional se basa en cuestiones ciertamente rentables, pero meramente coyunturales o incluso casuales; no se conseguirá a largo plazo, un cambio sustantivo en las condiciones de vida, salud y educación que los peruanos necesitamos.

La unidad entre la universidad, el Estado y la empresa que se propone desde la CTS, no es una receta mágica para el desarrollo, pero es una alternativa que ha producido resultados exitosos en diversos países como Estados Unidos, cuyas universidades a mediados del siglo XIX dan un importante salto, gracias al apoyo de empresarios como Ezra Cornell, Andrew Carnegie o John Rockefeller, que fundaron universidades o dieron apoyo económico a otras; de modo que están vinculados al despegue académico de universidades como Cornell, Harvard, Chicago, Clark, etc. (Greenwood, 2011).
Ciento cincuenta años después, el resultado es que, las universidades estadounidenses poseen un gran poder económico fruto de la investigación y las invenciones tecnológicas que generan. En el caso de la computación, el MIT por ejemplo, tuvo una importante participación en la creación de la computadora y el perfeccionamiento de diversos modelos cibernéticos. Hoy en día, el poder económico de estas universidades es tal que la Universidad de Harvard posee pozos petroleros en Kuwait y la Universidad de Austin en Texas, maneja un presupuesto anual mayor que Nicaragua.

Este nivel de desarrollo institucional les permite desenvolverse con autosuficiencia y presentarse como un buen modelo para otras universidades. Para muchas universidades latinas -las estatales al menos-, este modelo a seguir se topa con una visión paternalista de sus autoridades y de prácticamente toda la comunidad universitaria que las conforma, porque piensan que el Estado debe darles todo, y obvian que la universidad cuenta con capital humano y recursos suficientes para crecer y consolidarse por sus propios medios. ¿Acaso no congrega a las mentes más brillantes del país? ¿No son las universidades los repositorios del conocimiento de más alto nivel en el Perú?

Una sociedad del conocimiento, como señala Peter Drucker (2003), maximiza sus recursos a partir del conocimiento, de modo que no son las naciones que cuentan con mayores recursos naturales las que alcanzan mayor poder económico y calidad de vida, sino aquellas que aprovechan mejor su capital humano. Para muestra tenemos países como Japón o Luxemburgo que a pesar de contar con reducido territorio y limitados recursos naturales, poseen altos estándares de economía, salud y educación (Oppenheimer, 2008).

Pues bien, dado que muchas universidades no participan en la solución de los problemas del país, ya sean, sociales, económicos, viales, 
tecnológicos, psicológicos o educativos; es lógico cuestionar su existencia y plantear en estado de emergencia a la universidad peruana, pero antes es necesario analizar los factores que motivan esta crisis, no perdiendo de vista el trinomio investigación-enseñanza-extensión.

\section{LA CRISIS DE LA UNIVERSIDAD PERUANA}

Aunque suene dura esta sentencia, es real: la universidad peruana está en crisis. Esta crisis ha sido condicionada por diversos factores. Algunos de corte político, otros económicos, otros sociales, etc. Es decir que podemos identificar diversos agentes causales que han originado esta crisis y que bien pueden ser vistos como los más importantes actores en este escenario dispuesto en el terreno de la educación superior. Entre ellos tenemos el Estado, los profesores, los estudiantes y la sociedad.

En cuanto al Estado, podemos decir que ha propiciado esta situación cuando promulgó el D. L. 882 para la Promoción de la Inversión en la Educación, pues ese fue el germen que propició la proliferación de universidades que han distorsionado el verdadero sentido de esta institución, pero dado que tienen demanda, se siguen manteniendo en el mercado. Sin embargo esta demanda está sujeta a la mediocridad de algunos estudiantes y a las limitaciones de otros que desean estudiar en una buena universidad.

En el primer caso, muchos estudiantes desean terminar sus estudios rápido y de manera sencilla, por lo que optan por universidades que les convalidan sus estudios técnicos o que les exigen muy poco. Las business university brindan esa oferta y hay quienes la toman. En el segundo caso, estudiantes que no pueden acceder a una universidad de buen nivel, ya sea porque no cuentan con el dinero suficiente o porque no han tenido la preparación adecuada en el colegio que les permita ingresar, aprobando el examen, se resignan a estudiar en una universidad mercantilista como las que describimos.
Cabe señalar que no toda universidad privada es una universidad negocio, sino aquellas que no priorizan la investigación como parte de la formación y producción universitaria. De hecho, hoy en día muchas universidades estatales han seguido las fórmulas de las business university, a nivel de postgrado, ofertando maestrías y doctorados, carentes de las herramientas y exigencias sustanciales para hacer investigación. Solo cubren la demanda de algunos profesionales que buscan tener su título de maestría o de doctorado para acceder a un mejor puesto en el trabajo, sin considerar que como dice Arellano (2008) el doctor es "la locomotora de la ciencia, el creador de novedades para el desarrollo y el bienestar que viene con ella" (p. 37).

Hasta se ha incurrido en la figura de los "doctorados bamba" cuando se otorgó doctorados a profesores universitarios, considerando solo sus años de servicio y la presentación de una tesis doctoral que seguramente, no presenta ningún aporte a la ciencia. Así pues, un doctor es aquel que hace ciencia y que hace su trabajo con sólidas bases científicas. En nuestro país en cambio abundan los doctores de "etiqueta" o "bamba".

Lo que no consideran las universidades que endosan estos "doctores" a la sociedad, es que las naciones que no tienen "doctores de verdad" están condenadas a ser recipientes pasivos de lo que hacen otros países. En palabras de Arellano, "un país que no hace ciencia nunca podrá ser líder en el mundo moderno" (Arellano, 2008, p. 38).

El Estado tiene parte de responsabilidad en esta cuestión, por avalar este tipo de actividades, aunque esté de por medio la autonomía universitaria, que si bien es fundamental para el crecimiento de las universidades, cuando es utilizada de pretexto para apañar estas barbaridades, no puede ser un medio de defensa, sino muy por el contrario, solo es una artimaña legal para despercudirse de la responsabilidad 
que se tiene frente a todo un país de no cumplir con el rol verdadero de estas casas de estudios.

En ese sentido, Lovón (1996) afirma que "sin la existencia de una normalegal, las declaraciones de principios, o los fines expuestos en los estatutos de las universidades, sólo serían manifestaciones de buena voluntad, pero se requiere la existencia de una norma legal imperativa, que obligue a realizar las acciones de control de la calidad de la enseñanza universitaria" (pp. 80-81). Ello no significa perder su autonomía, sino simplemente garantizar que la universidad cumple su papel como le toca al ser una de las instituciones más emblemáticas de la sociedad.

Por otro lado, no puede obviarse el hecho de que el Estado peruano no ha brindado el apoyo económico que la investigación científica y el desarrollo tecnológico requieren. El Estado peruano solo aporta un 3\% del PBI a las universidades nacionales del país (Delgado y Alcázar, 2004). La situación en otros países de Latinoamérica es similar: México destina el 4,4\% de su PBI, Chile 4,2\% y Argentina 4\%. Vale decir todavía, que casi todo el gasto educativo se va en pagar salarios. Esta cifra es del 95\% en México, del 90\% en Brasil y del 80\% en Argentina (Oppenheimer, 2008). Es decir que la ayuda del Estado no permite hacer investigación. Pero no olvidemos que la producción científica puede ser llevada a cabo por fondos concursables.

En la actualidad hay diversos concursos de investigación del Concytec, CIES, Preal, etc., que subvencionan investigaciones de tipo social, tecnológico, educativo, económico, o de salud; de manera que para las universidades que no cuentan con fondos propios para investigación, no hay excusas para no hacer investigación científica. Eso obviamente no exime al Estado de su responsabilidad para con las universidades, ya que se debe aumentar el presupuesto y la inversión educativa a nivel superior; considerando que el Perú invierte 2,344 USD al año por estudiante universitario, mientras que Brasil invierte 10,306,
Chile 7,71l, México 4,331, Argentina 3,047, EEUU 19,802, Canadá 14,579, Suecia 13,224 y España 5,038 (Aranzamendi, 2008).

Pero aumentar el presupuesto a las universidades estatales supone también exigir más de ellas. En ese sentido, toca a los profesores universitarios liderar los procesos de transformación social. Claro que difícilmente podrán llevarse a cabo si no hacen investigación. En ese sentido hay que decir, que son muy pocos los profesores universitarios del país que hacen investigación científica o tecnológica. Este caso es más alarmante en las universidades públicas y/o en aquellas que se encuentran en provincias, muy a pesar de que los profesores de las universidades nacionales reciben un pago por investigación. Que no investiguen entonces, no es ya solamente una cuestión de laxitud o incapacidad, sino una falta moral, porque están cobrando un sueldo por algo que no hacen, sueldo que además proviene del bolsillo de todos los peruanos.

Así pues, el otro componente de la crisis universitaria -y quizá el más importantees la falta de profesores académicamente preparados con la capacidad y la vocación de gestionar eficientemente el conocimiento a través de la investigación. Ciertamente muchos profesores, aducen que el sueldo que les pagan es insuficiente para investigar, pero repetimos que pueden participar de concursos nacionales e internacionales de investigación, además de solicitar apoyo de las empresas privadas como se trabaja en el modelo CTS. Sin embargo, creemos que si quisieran podrían hacer más con el mismo sueldo que reciben (Arias, 2003).

En realidad lo que ocurre es que su trabajo en la docencia universitaria, no está amparado por una firme vocación de enseñar ni de investigar, sino que su principal motivación ha sido o es, ganar dinero y tener la estabilidad que proporciona el trabajar para el Estado, aunque no se cumpla con las demandas sociales que tiene todo trabajador público. Esto es así, porque en muchas de las 
universidades públicas y privadas del país, los profesores ingresan al claustro universitario gracias a favores políticos, amistad o nepotismo. Es decir que no existe un adecuado proceso de selección de los profesores que ingresan a trabajar a la universidad. Esto ha generado que algunas universidades estén a la zaga de la frontera del conocimiento, y que por ende, la producción académica en el Perú sea muy limitada.

Por ejemplo, según el Science Citation Index Perú tenía para 1997, 173 publicaciones registradas, Colombia 545, Chile 1770 y España 22,077 (Aranzamendi, 2008). Si consideramos que la producción académica se concentra mayoritariamente en la capital, esto deja todavía peor a las universidades de provincia. Un profesor promedio de Lima cuenta en su haber con aproximadamente 30 artículos publicados, mientras que un investigador top tiene en promedio 200.

En Arequipa, un investigador top tiene en promedio 20 investigaciones, y muchas de ellas incluso, publicadas solamente en medio locales o en revistas no arbitradas, mientras que sus pares de Lima publican en revistas indexadas. La producción científica también es mayor en Lima con respecto a las revistas de investigación y los libros que se publican. Por todo ello, es que podemos decir que prácticamente la producción académica nacional se circunscribe a Lima.

En resumen, no se puede soslayar el hecho de que los profesores universitarios deben tener mayor producción académica y estar preparados para generarla, de no ser así, aunque sean excelentes profesionales, la universidad no es el lugar más adecuado para ellos, ya que si no investigan tampoco podrán ser buenos profesores.

Precisamente, otro aspecto que ha sido también motivo de análisis dentro del marco del tema que nos ocupa, es que los profesores de este nivel de enseñanza, no han sido formados como profesores, sino que son profesionales con cierta trayectoria y experiencia en un determinado campo profesional que se han sumado (en algunos casos, posiblemente por las razones antes señaladas), al cuerpo docente universitario. En ese sentido, la docencia universitaria, dispone de estrategias didácticas muy particulares, que difieren de las empleadas en el nivel escolar (Castilla, 2000). Ignorarlas podría ser efectivamente, una causa de la deficiente formación que reciben los estudiantes universitarios en el país. En consecuencia, a los profesores universitarios les hace falta mayor capacitación pedagógica y formación especializada en docencia universitaria.

Pero, dada la estrecha vinculación entre la enseñanza y la investigación en la universidad, pensamosqueademás delaformaciónpedagógica, un profesor que no investiga, difícilmente podrá enseñar bien, ya que su grado de rigor académico en algunos temas será limitado, seguramente no estará actualizado con los últimos avances científicos de su campo y no podrá transmitir al estudiante el entusiasmo por profundizar en el curso que enseña, ya que él mismo no lo hace.

En ese sentido, en Arequipa, muchos de los profesores universitarios se encuentran tan desfasados queenseñan temas de la primera mitad del siglo pasado e ignoran los últimos alcances científicos en sus propias áreas de conocimiento. Por ejemplo, en 1996 se realizó una encuesta en la ciudad de Arequipa que reportó que el 73\% de estudiantes de la Universidad Nacional de San Agustín y 67\% de la Universidad Católica de Santa María pensaba que sus profesores "no domina los cursos que enseñaba". El 11\% de estudiantes de la UNSA y el 10\% de estudiantes de la UCSM señalaba que sus profesores se encontraban "totalmente desactualizados" (Centro de Estudios e Investigaciones Filosóficas Universitas, 1996).

A modo de conclusión sobre este aspecto, podemos decir que los profesores universitarios deben tener un perfil caracterizado por las siguientes cualidades: grados y títulos especializados, formación pedagógica, 
experiencia profesional, inquietud académica, actitud crítica, conocimientos actualizados y especializados, capacidad de investigación, claridad epistemológica, proactividad, personalidad estable, creatividad, publicaciones, membresía en sociedades académicas nacionales e internacionales, sentido ético y sólidos valores morales (Knight, 2009).

En la medida que los profesores universitarios cumplan con estos requisitos, su espacio en la educación superior estará plenamente justificado y podrán contribuir con el desarrollo del país, a través de la generación de conocimiento mediado por la investigación científica, además de la formación que dan a los jóvenes estudiantes que acuden a la universidad.

En este punto, toca ya hablar de los estudiantes universitarios, que como hemos adelantado, en muchos casos no optan por la universidad, por las razones adecuadas o con la claridad necesaria para estudiar como se debe mientras transitan por los claustros universitarios.

De hecho, si bien son muchos los que ingresan a la universidad, son pocos los que terminan. En Argentina por ejemplo, egresan dos de cada diez estudiantes y en México solo el 30\% termina su carrera (Oppenheimer, 2008). En ese sentido, nos atrevemos a decir que buena parte de los estudiantes universitarios lleva sus estudios mediocremente, pero esto tiene diversas implicancias que es importante analizar. Por un lado, las deficiencias académicas de los estudiantes universitarios se deben a que en el colegio no han sido formados adecuadamente.

Por ejemplo, en secundaria el 68\% de estudiantes peruanos no comprende lo que lee, y el $41 \%$ apenas puede resolver problemas matemáticos simples. Esto se debe a su vez, a que aunque se dice lo contrario, no se da la debida importancia a la educación, ya que existe un divorcio entre lo que se predica y lo que se hace. Para muestra, considere que si bien el gobierno peruano exige 1100 horas de enseñanza en primaria y 1200 en secundaria al año, en las zonas rurales se llega a las justas a 250 horas de enseñanza. La consecuencia es que existen brechas muy grandes entre la enseñanza urbana y la rural, encontrándose en seria desventaja los estudiantes que provienen de las zonas altoandinas.

A nivel nacional por ejemplo, el analfabetismo es de $12 \%$, pero dos tercios están en la zona rural y $76 \%$ de este sector lo conforman las mujeres. Si nos comparamos con otros países, veremos que en el Perú se trabajan 22 semanas de clases reales en lugar de las 36 planificadas, en Brasil, Chile y Colombia se trabajan en cambio 40 semanas de clase (Aranzamendi, 2008).

Otro aspecto es que no se hace una selección adecuada de estudiantes, pues los exámenes de ingreso solo valoran los conocimientos de los estudiantes, pero no su disposición psicológica hacia la carrera. Tampoco se filtra adecuadamente a los estudiantes, sobre todo en las universidades nacionales, donde los discentes pueden desaprobar los cursos tantas veces como su incapacidad se los permita, sin considerar que están estudiando con el dinero de los peruanos, y el mostrar un bajo rendimiento, desaprobar cursos o reprobar el semestre; implica que se está utilizando mal los fondos del Estado.

No se puede perder de vista que la educación estatal funciona bajo el esquema de una inversión que hace el Estado en los estudiantes y que luego se debe revertir en la sociedad, a través de su trabajo altamente cualificado y orientado a resolver los problemas sociales de la población. Pero si los estudiantes no rinden como se debe o se retiran, la empresa educativa en la universidad no cumple su cometido, sino que por el contrario, hecha millones de soles al agua. A esto se suma que tampoco se hacen filtros económicos adecuados, pues muchos de los estudiantes que asisten a las universidades estatales pueden pagar sus estudios en una universidad privada. Esta situación es recurrente en varios países de América Latina. Por ejemplo en Brasil, México y Argentina, el 70\% de estudiantes universitarios 
pertenece al 20\% más rico de la población (Oppenheimer, 2008). Todo ello significa que no se hace un gasto eficiente con los recursos del Estado.

Por otro lado, si a esto se agrega que el otro elemento de la ecuación universitaria, vale decir el profesor, no hace lo que le toca como tal, el resultado será indefectiblemente, una enseñanza de muy bajo nivel académico que poco puede aportar a la sociedad. Así, los engranajes de la maquinaria universitaria están tan desgastados que es muy difícil echar a andar la universidad sobre los rieles de la investigación, la enseñanza y la extensión.

Lo lamentable de todo este asunto es que esta situación, pasa desapercibida para la comunidad, ya que en encuestas recientes, 37\% la población arequipeña señala que la UNSA es la mejor universidad de la ciudad (Aurum, 2012), desconociéndose que en esta universidad la investigación ha dejado de ser su motor, ya que no solo no cuentan con revistas especializadas e indexadas, sino que la mayoría de sus profesores no investigan ni cuentan con producción académica relevante. Esta situación se ve opacada por los casi 200 años de existencia de la universidad, y por la tradición que ha tenido en décadas anteriores, cuando efectivamente, la UNSA era un foco de cultura y conocimiento.

De esto, empero, queda solo el recuerdo. A los padres de familia poco les importa esta penosa situación, ya que solo demandan de la universidad que haga profesionales a sus hijos, pero ignoran que la profesionalidad que deviene de la formación universitaria se sustenta en el trabajo científico de los profesores y los estudiantes. De hecho, los propios padres no brindan los soportes adecuados para la educación de la prole, ya que solo el $0.1 \%$ de la población destina presupuesto para comprar libros (Aranzamendi, 2008). Sin libros qué puede hacer un estudiante universitario, aunque disponga de otros elementos como el Internet.
Lo que ha ocurrido en términos sociales es que se ha instrumentalizado el conocimiento, de modo que se han perdido las capacidades críticas y de reflexión sobre cuestiones sumarias como la importancia del conocimiento y sus vinculaciones con el destino del país. Destino que en gran medida, reposa en la educación, la investigación y las jóvenes generaciones; ingredientes todos estos que confluyen en la universidad, por eso le toca jugar un rol protagónico en el desarrollo del Perú.

Como dicen Roa y Suárez (2006) "la educación superior puede apostarle nuevamente a ser filtro, convertido en conciencia crítica, en lo que sucede en la realidad" (p. 128), ya que si bien la educación es la base del desarrollo que sostiene la sociedad, para que la educación universitaria esté a la altura de las demandas sociales del país, debe pasar del paradigma de enseñar al de aprender, sí, aprender de los modelos de gestión del conocimiento de las universidades más exitosas del mundo.

Un ejemplo próximo a seguir, podría ser Brasil, donde las universidades son responsables del 90\% de la producción científica del país y de cada 100 jóvenes solo 20 van a la universidad, porque no todos tienen la misma vocación por el estudio (Aranzamendi, 2008). Deberá también brindar ofertas profesionales de acuerdo a los tiempos presentes, las nuevas especialidades y las necesidades sociales.

Se debe ampliar su infraestructura física y virtual, así como su visión de universidad, de modo que la formación universitaria revalore las capacidades intelectuales que favorecen la investigación y la reflexión crítica. Se debe instaurar un nuevo orden ético, donde los méritos orienten los procesos de mejora y promoción en la universidad, donde se enfaticen las cualidades afectivas, sociales y emocionales que se cultivan al ser persona (Ruíz, 2013). En suma, se trata de que la universidad responda a su verdadera naturaleza, a sus fines intrínsecos. 
Conseguir esto requiere, empero, de que en las universidades se tome conciencia de la identidad que tienen como repositorios del saber primero y como empresas después, pero ambas son trascendentales para definir el norte que se quiere seguir. Por ello es fundamental identificar los rasgos propios de cada universidad, más allá de las características comunes. Estos rasgos constituyen su sello institucional y su identidad.

\section{IDENTIDAD DE LA UNIVERSIDAD: EL CASO DE LAS UNIVERSIDADES CATÓLICAS}

Podemos decir que las universidades de Latinoamérica comparten características que en los últimos años se han hecho más evidentes. Por un lado, está la crisis que es común a varias universidades de Latinoamérica (escasa investigación, deficiencias en la enseñanza, profesores sin mérito académico, limitada inversión estatal en la educación superior, estudiantes sin vocación y con malos hábitos de estudio, equipamiento e infraestructura insuficiente, etc.), pero por otro, también se distinguen ciertas características demográficas extendidas entre las universidades de los países de la patria chica, como son el acceso limitado a la universidad, la feminización de la educación superior y la explosión de universidades privadas (Beneitone, Esquetini, González, Maletá, Siufi y Wagenaar, 2007).

Todos estos factores han determinado una identidad universitaria que se aleja de los valores originarios de la universidad, ofreciéndonos un conocimiento pragmático, relativizado y desconectado de la sociedad. En ese sentido, cada universidad como toda institución responde a una identidad.

Entre los modelos de universidad podemos distinguir los siguientes: a) un modelo romántico, que implica el cultivo de ideales en pos de una sociedad libre, justa y fraterna; b) en modelo republicano, que responde a un ideal ilustradoracionalista; c) el modelo emancipacionista, que propende a la educación para la emancipación dentro de un marco ideológico marxista; y d) el modelo católico, que es fiel a la identidad y los principios de la Iglesia Católica.

Cada modelo ha tenido un momento de protagonismo en el tiempo. El modelo romántico con la revolución francesa y el liberalismo europeo que se focalizó en Francia e Inglaterra. El modelo republicano que se empieza a institucionalizar durante la Ilustración, y más fuertemente, a partir la reforma napoleónica y la reforma alemana. El modelo emancipacionista hizo eco de la teoría marxista y su interpretación de la sociedad, del cual se derivó un modelo de enseñanza universitaria que reacciona frente a las desigualdades. La reforma de Córdova que tuvo lugar en Latinoamérica responde a este modelo. Sin embargo, el modelo emancipacionista se moderó con el influjo de los teóricos de Frankfurt y el enfoque sociocrítico, que se muestra más crítico con el propio marxismo.

Finalmente, el modelo católico fue el que dio origen a la universidad en el siglo X, pero con el correr de los años y el proceso de secularización, las universidades católicas perdieron su identidad y asumieron otros modelos, unos más academicistas, otros más reaccionarios, otros más idealistas y otros más mercantilistas. Sin embargo en el siglo XIX, con el Cardenal John Henry Newman se intentó restablecer el modelo católico que se estaba perdiendo. Su labor se centró en universidades de Irlanda y Bélgica, con un discurso que armoniza la fe y la razón (Newman, 1993). Luego, en 1990, el Sumo Sacerdote Juan Pablo II, escribe la Constitución Apostólica Ex Corde Ecclesiae, en la que explica los principios que deben regir las universidades católicas y desarrolla sus orientaciones fundamentales, que tienen un carácter normativo.

La Ex Corde Ecclesiae es así, la carta magna de las universidades católicas. En ella se insta a defender los valores de la fe católica desde las aulas universitarias, a través de la investigación, la enseñanza y la formación de los estudiantes 
que libremente reunidos con sus maestros, son animados por el amor del saber (Juan Pablo II, 1990, p. 5).

Se parte, como hiciera el Cardenal John Newman, por reconocer que la universidad es una empresa eminentemente intelectual. De hecho Newman distingue las verdaderas universidades de las escuelas profesionalizantes, es decir aquellas que solo buscan formar profesionales. En ese sentido el aporte de Newman trasciende a lo pedagógico y se incrusta en el corazón de la universidad con una profunda visión filosófica (Athié, 2012).

En otras palabras el amor por el saber y la consecuente búsqueda de la verdad es lo que define a las universidades católicas del mundo. Por ello, la investigación, en sus diversas formas teórica, aplicada, experimental, etc.--, es también la vía que nutre su naturaleza, pero además su identidad como institución católica, señala cuatro preceptos por los que debe transitar la investigación.

Según la Ex Corde Ecclesiae la investigación científica debe reposar en la integración del saber, el diálogo entre la fe y la razón, la responsabilidad ética y una perspectiva teológica. Estos son los rasgos que caracterizan la investigación en las universidades católicas y que no buscan sino, derrocar la visión fragmentada del hombre que ha sido impuesta por el positivismo y el racionalismo, ambas posturas dominantes en el quehacer científico hodierno.

Así pues, no es que la fe se opone a la ciencia, ya que ambas son "dos alas con las que el espíritu humano se eleva a la contemplación de la verdad" (Juan Pablo II, 1998, p. 7). A lo que se opone la fe es al "cientificismo" que con una visión reduccionista limita el campo de estudio de los saberes humanos, a los puramente experimentales, cuantificables y observables; aun cuando muchos fenómenos de la realidad son eminentemente cualitativos, metafísicos y sobrenaturales.
El papel de la investigación en las universidades católicas es aproximarse a la realidad a través de una honda reflexión antropológica y alto rigor académico, para restaurar el conocimiento escindido y lograr una síntesis integradora que forje una cultura reconciliada y esté al servicio de toda la humanidad. Ello se encuentra en viva conexión con las funciones de extensión y enseñanza de la universidad, y a través de su reformulación, da respuesta a la emergencia educativa de los tiempos actuales, desde una reflexión de la verdad fundada en Cristo, ya que "no hay verdadera educación sin una concepción de verdad" (Carriquiry, 2012, pp. 42-43).

En ese sentido, las universidades católicas están llamadas no solo a formar académicos y profesionales, sino personas íntegras con una sólida visión del mundo a partir de la unidad fe-razón. Así pues, no pueden existir contradicciones entre la fe y la razón, cuando se acompañan de manera equilibrada. Sus excesos serían nocivos para la universidad, ya que por un lado el inmanentismo y por otro el fideísmo, agotarían su misión contemplativa y terminarían también desvirtuando sus fines.

Es decir que "una universidad católica no existe ante todo para circunscribir un ámbito de difusión de la fe, aunque esa dimensión no sea ajena a su vida" (García, 2004, p. 117). Se trata de que la universidad sea un foco de cultura que integre todo el conocimiento, en un horizonte que se amplía y enriquece con la adopción de su real dimensión a través de la fe.

La cultura es pues repositorio de la fe, y por ser inseparable de la vida del hombre y expresión de la vida humana (Ferrogiaro, 2012), toda la universidad gravita en torno a ella. Por eso, la universidad está llamada a ser un instrumento cada vez más eficaz del progreso cultural, tanto de las personas como de la misma sociedad (García, 2003). 
De hecho, como dice Morandé (2012) la universidad está al servicio de la sociedad, he ahí su función de extensión. Más aún, si restablecer la cultura significa hacer frente al nihilismo y al relativismo que se ha esparcido en la sociedad y ha tocado a no pocas universidades católicas.

En ese sentido, la transmisión de la fe en la universidad católica, no puede ser una mera sentencia y mucho menos contradecir el modelo de vida de la Iglesia Católica. Ella debe preparar el ámbito para el despliegue de las potencialidades humanas de todos los miembros de la comunidad universitaria, profesores, alumnos y administrativos; y de la sociedad misma.

La espiritualidad católica debe ser cultivada y promovida en todos estos niveles, que engranan las funciones de la universidad y son travesados por el respeto a la persona humana; que para el caso de los trabajadores se plasma en la promoción de su bienestar, a los profesores les corresponde ser apoyados en su tarea de integrar el pensamiento cristiano con las ciencias modernas, y a los estudiantes el de brindarles una formación integral en conocimientos, capacidades y valores católicos. Todos en la comunidad universitaria cumplen su rol y trabajan juntos por la noble misión de elevar el espíritu del hombre y contribuir con el desarrollo de una cultura de vida, de paz y amor. Se trata entonces de dar testimonio institucional de la vida de Cristo.

De ahí, que la identidad de la Iglesia Católica ilumina la vida institucional de la universidad, que como empresa que es expone una misión que determina su estructura y sus recursos en función de su visión. Como universitas, tanto profesores como estudiantes colaboran en el proceso de búsqueda de la verdad y de ella emanan sus funciones de enseñanza y extensión.
Una universidad católica entonces, tiene una identidad que deviene de la Iglesia Católica y que la define como institución académica y como organización de personas Esta identidad, la diferencia además, de otras instituciones y hasta de otras universidades que tienen otras prioridades.

Enconclusión, podemos decirquelainvestigación en la universidad católica busca la verdad a través de la integración del saber, la enseñanza está orientada a la formación integral de la persona en un diálogo fecundo entre la fe y la razón, y la extensión se manifiesta en la evangelización de la cultura que cierra el círculo virtuoso de su identidad. Una universidad católica debe reflejar así, la integración de la ciencia con la fe, tanto en los contenidos de la enseñanza como en las manifestaciones en el aprendizaje. Debe motivar el espíritu investigativo de los profesores y los estudiantes, quienes han de ser formados para autorregular las capacidades que les permitan transformar el mundo en un orbe más humano. Deben ser formados por tanto, para el encuentro con otros y consigo mismos, a través de la mediación de cualidades personales y valores éticos (Roa y Suárez, 2006).

En resumen, la crisis que vive la universidad actual se debe a dos cuestiones: por un lado, se ha olvidado la verdadera naturaleza de la universidad y por otro, muchas universidades se han fundado sin tener claro cuál es su identidad. Aunque ambas cuestiones se encuentran relacionadas, la primera pospone los fines de la universidad como academia y la segunda atenta contra sus principios como organización. El resultado es empero, siempre el mismo: una institución aletargada que no es capaz de brindar soluciones concretas a los problemas sociales más álgidos, una institución desmoralizada que busca respuestas a interrogantes no formuladas, esta institución solía llamarse universidad. 


\section{REFERENCIAS}

Aranzamendi, L. (2008). Crisis universitaria. Arequipa: ADRUS.

Arellano, R. (2008). Bueno, bonito y barato. Lima: Planeta.

Arenas, M. (1996). El Dean Valdivia. Símbolo de la Arequipa Republicana. Arequipa: s/e.

Arias, W. L. (2003). La enseñanza en la universidad. Entre Claustros, 1(1), 13-16.

Athié, R. (2012). Aportaciones del cardenal Newman a las Universidades Católicas. Persona y Cultura.

Aurum. (2012). ¿Cuál es la mejor universidad de Arequipa? Arequipa Empresarial, 18, 40

Benavides, M. y Etesse, M. (2012). Movilidad educativa intergeneracional, educación superior movilidad social en el Perú: Evidencias recientes a partir de encuestas a hogares. En R. Cuenca, Educación Superior. Movilidad social e identidad. (pp. 51-92). Lima: IEP.

Beneitone, P.; Esquetini, C.; González, J.; Maletá, M.; Siufi, G. y Wagenaar. R. (2007). Reflexiones y perspectivas de la educación superior en América Latina. Informe final - Proyecto Tuning - América latina 2004-2007. s/l/e: EuropeAid.

Bunge, M. (1981). Ciencia y desarrollo. México: Siglo XX.

Cardó, A. (2005). Iglesia y educación en el Perú. Arequipa: UCSP.

Carriquiry, G. (2012). Desafíos y tareas de la Universidad Católica a la luz de la emergencia educativa. Persona y Cultura, 10(10), 39-65.

Casales, J. C. (2005). Determinantes de la eficacia directiva en una institución universitaria. Revista Cubana de Psicología, 22(1), 42-55.

Castilla, E. (2000). Didáctica universitaria. Lima: San Marcos.

Castro, J. F.; Casavilca, P. y Lizarzaburu, R. (2010) Crecimiento económico y demanda por educación superior en el Perú: un estudio para el periodo 2004 2006. Apuntes, 66(1), 29-58.

Castro, R. J. y Valdivia, J. (2012). Propuesta metodológica de un Sistema integral de Gestión Universitario. Revista de Administración de Negocios, 1(1), 73-92.

Centro de Estudios e Investigaciones Filosóficas Universitas. (1996). La calidad educativa en la universidad. Philosophica, 6, 5-16.

Céspedes, V.S. (1994). La cultura de la vida y la universidad. Philosophica, 5, 4-7.

Delgado, F. y Alcázar, M. (2004). Informe sobre educación superior universitaria. Lima: ANR.
Drucker, P. (2003). El management del futuro. Buenos Aires: Editorial Sudamericana.

Ferro, J. (2001). La globalización y el conocimiento. Reflexiones acerca del impacto de la modernización global sobre el desarrollo de los currícula académicos. Zona Próxima, 2(1), 24-29.

Ferrogiaro, L. (2012). La Universidad Católica y su misión en la promoción de una cultura humana y cristiana. Persona y Cultura, 10(10), 66-82.

García, A. (2003). El desafío cultural de las universidades católicas, consideraciones en torno a Ex Corde Ecclesiae. Persona y Cultura, 2(2), 65-87.

García, A. (2004). La fe cristiana en la universidad. Persona y Cultura, 3(3), 104-126.

Greenwood, J. (2011). Historia de la psicología. Un enfoque conceptual. México: McGraw-Hill.

Hubeñak, F. (2008). Historia integral de occidente desde un enfoque cristiano. Argentina: EDUCA.

Juan Pablo II (1990). Constitución Apostólica Ex Corde Ecclesiae. Lima: Epiconsa.

Juan Pablo II (1998). Documento Pontificio Fideset Ratio. Lima: Epiconsa.

Knight, P. T. (2009). El profesorado de educación superior. Formación para la excelencia. Madrid: Narcea.

Lazo, J. (2002). Metodología del trabajo universitario. Lima: UIGV.

Lovón, A. (1996). La educación y la ley. Philosophica, 6, 7681.

Molina, M. M. (2008). Introducción al estudio de la universidad en Latinoamérica. Revista Latinoamericana de Estudios Educativos, 4(1), 129-142.

Morandé, P. (2012). La vocación de servicio de la universidad a las personas y la sociedad. XX años de vigencia de Ex Corde Ecclesiae. Persona y Cultura, $10(10), 12-22$.

Newman, J. H. (1993). La fe y la razón. Sermones universitarios. Madrid: Ediciones Encuentro.

Oppenheimer, A. (2008). Cuentos chinos. El engaño de Washington, la mentira populista y la esperanza de América Latina. Buenos Aires: Editorial Sudamericana.

Perkins, J. A. (1967). La universidad en transición. México: Uteha.

Piscoya, L. (2006). Ranking Universitario en el Perú. Lima: ANR. 
Quintero, C. A. (2010). Enfoque Ciencia, Tecnología y Sociedad (CTS): perspectivas educativas para Colombia. Zona Próxima, 12(1), 222-239.

Reynoso, J. (1998). Formación académica profesional científicay humanista en la universidad. Arequipa: UNSA.

Roa, A. y Suárez, J. R. (2006). La sociedad globalizada y el papel de la educación superior. Zona Próxima, 7(2), 108-131.
Ruíz, M. F. (2013). El futuro de la educación (superior). Una reflexión entre la doxa y la episteme. Educación, 22(42), 7-27.

Sime, L. (2001). Universidad y currículo. Construyendo el cambio. Lima: PUCP. 